$\xi=-1$

\title{
A Novel Look Back $N$ Feature Approach towards Prediction of Crude Oil Price
}

\author{
Rudra Kalyan Nayak ${ }^{1 *}$, Kuhoo $^{2}$, Debahuti Mishra ${ }^{3}$, Amiya Kumar Rath ${ }^{4}$, Ramamani Tripathy $^{5}$ \\ ${ }^{1,3,5}$ Dept. of Computer Sc. \& Engineering, Institute of Technical Education and Research, Siksha O Anusandhan (Deemed to be) \\ University, Bhubaneswar, Odisha, India \\ ${ }^{2}$ Department of Mechanical Engineering, College of Engineering and Technology, Bhubaneswar, Odisha, India \\ ${ }^{4}$ Dept. of Computer Sc. \& Engineering, Veer Surendra Sai University of Technology, Burla, Sambalpur, Odisha, India \\ * Corresponding author E-mail: rudrakalyannayak@gmail.com
}

\begin{abstract}
Prediction of crude oil prices in advance can play a significant role in the global economy. Change in crude oil price affect wide range of application for economic and risk projection. Crude oil price forecasting is a challenging task due to its complex nonlinear and chaotic behavior. During the last decade's researcher have designed many classification algorithm for crude oil prediction. The major challenge for any unsupervised dataset is to define a class label for every feature of its dataset. This paper, propose a new novel technique, look back $N$ feature (LBNF) algorithm to discover class label. Later the classifier support vector machine (SVM) with $k$-nearest neighbor $(k$ $\mathrm{NN}$ ) has been used to classify the current feature vector to predict the crude indices one day, one weak, one month in advance. We have checked our algorithm with standard recent MCX INR Daily and CFD USD Real Time crude oil dataset. To prove the effectiveness of proposed algorithm we have compared it with recent Grey wave forecasting method and the experimental result is found to be better than this method.
\end{abstract}

Keywords: Support Vector Machine (SVM), k-nearest neighbor (k-NN), Grey wave forecasting method, autoregressive integrated moving average (ARIMA), Look Back N Feature (LBNF).

\section{Introduction}

From the earth we get varieties of Natural resources. These natural resources are categorized into two parts: one is biotic and another one is abiotic. Biotic resources include plants, animals, and fossil fuels. The three fossil fuels are coal, oil, and natural gas. Crude oil is a mixture of naturally occurring; unrefined petroleum product composed of hydrocarbon deposits and is typically obtained from oil drilling. Today, the world's economy is largely dependent on fossil fuels. The United States, Saudi Arabia, and Russia are the leading producers of oil in the world [1-2]. Among all natural resources crude oil plays an important role in our global economy. Forecasting of crude oil price is a very challenging factor for individuals, governments and industries due to instability of oil prices. Disparity of Oil price provides direct impact on the Indian economy as well as the communities. To reduce the negative impact of the price variation it is necessary to forecast the oil price. Now a day's main focus of all researchers is to solve the problem of fluctuating crude oil prices with high accuracy. For oil price prediction, numerous machine learning methods were proposed such as artificial neural networks (ANN) [3-14], and support vector machine (SVM) [15-18]. These are nonlinear models which may produce more accurate predictions if the oil price data are strongly nonlinear [19].

Lean Yu et al. [20] introduced a novel decomposition model using artificial intelligent (AI) technique [21] of extended extreme learning machine (EELM) for model formation. The main purpose of this paper is to improve the performance of the model and is compared with other forecasting tools and similar ensemble learning paradigms to enhance the accuracy, reduce the time need for prediction of crude oil price. For prediction of crude oil price, Niaz Bashiri Behmiri et al. [22] surveyed varieties of methods like traditional, statistical and econometric techniques such as linear regression, random walk (RW), autoregressive integrated moving average (ARIMA), generalized auto regressive conditional heteroskedasticity (GARCH) etc. RW is taken as the scale by W. W. He Angela et al., P. Ekaterini et al. and M. Atilim et al.[23-25] to predict crude oil price and also L. Yu et al., Y. Lean et al., H. Kaijian et al. and L. Ziran et al. [20,26-28] chose ARIMA as benchmark for prediction of crude oil price.

Yanhui chen et.al [29] proposed a flexible graphical prediction method based on grey wave forecasting to forecast multistep ahead crude oil price. Authors compare their model with traditional time series method and also emphasise on weight computation complexity against both Akaike Information Criteria (AIC) and Bayesian Information Criteria (BIC). As a result they conclude that grey wave forecasting technique improved the forecasting accuracy for single and multistep ahead crude oil price prediction.

Our study investigates the prediction of crude oil using look back $N$ feature (LBNF) algorithm to discover class label and most of the classifiers SVM with $k$-NN [30] is used to classify the current feature vector. The objective of this paper is to generate crude indices of one day, one week, one month in advance. To prove the strength of our proposed algorithm it is compared with recent grey wave forecasting method [29] and ARMA $(1,1)$ model taking the standard recent MCX INR Daily and CFD USD real time crude oil dataset. The simulation result indicates that the proposed method 
has lower RMSE than grey wave forecasting and ARMA $(1,1)$ model and proved to be proficient model.

This paper is organized as follows: section 2 contains data set description, technical indicators, building of dataset, defining the class label, theoretical knowledge on SVM and $k$-NN as materials and methods. Section 3 deals with proposed architecture Experimental evaluation and result analysis is depicted in section 4. At last section 5 summarizes the paper along with this directs towards potential future work.

\section{Materials and Methods}

\subsection{Data Set Description}

Two recent crude oil datasets MCX INR Daily and CFD USD Real Time is collected from [31] http://in.investing.com/commodities/crude-oil spanning from $12^{\text {th }}$ December 2011 to $1^{\text {st }}$ August 2016 and $1^{\text {st }}$ July 2011 to $1^{\text {st }}$ August 2016 respectively out of which MCX INR Daily is in Indian rupees and other is in American dollar. Similarly two other datasets BRENT and WTI [32-34] are collected from https://fred.stlouisfed.org/series spanning from 20th May 1987 to 29th August 2016 and 2nd January 1986 to 29th August 2016 respectively, the description of the dataset can be found in http://www.eia.gov/dnav/pet/TblDefs/pet_pri_spt_tbldef2.asp.

Table 1 shows the statistics of all four datasets. Table shows that the dataset is too complex as the deviation from low value to high value is large for all the four datasets. Figure 1 shows the opening and closing indices of four datasets. It can be noticed that crude oil price has never been stable throughout the day.

Table 1: Crude Oil Dataset

\begin{tabular}{|l|l|l|l|l|l|l|l|}
\hline Crude Oil Dataset & Range in dates & $\begin{array}{l}\text { Number of } \\
\text { Features }\end{array}$ & $\begin{array}{l}\text { Highest } \\
\text { Value }\end{array}$ & $\begin{array}{l}\text { Lowest } \\
\text { value }\end{array}$ & Mean & Median & $\begin{array}{l}\text { Standard } \\
\text { deviations }\end{array}$ \\
\hline MCX INR Daily & $\begin{array}{l}\text { 12-Dec-2011 to 01-Aug- } \\
\text { 2016 }\end{array}$ & 1310 & 7507 & 1844 & 4698.7 & 5031 & 1309.61 \\
\hline $\begin{array}{l}\text { CFD USD Real } \\
\text { Time }\end{array}$ & $\begin{array}{l}\text {-Jul-2011 to 1-Aug- } \\
\text { 2016 }\end{array}$ & 1333 & 110.53 & 26.21 & 78.48 & 90.76 & 24.65 \\
\hline BRENT & $\begin{array}{l}\text { 20-May-1987 to 29- } \\
\text { Aug-2016 }\end{array}$ & 7639 & 143.95 & 9.1 & 43.26 & 26.75 & 34.11 \\
\hline WTI & $\begin{array}{l}\text { 2-Jan-1986 to 29-Aug- } \\
\text { 2016 }\end{array}$ & 7998 & 145.31 & 10.25 & 42.81 & 27.73 & 30.33 \\
\hline
\end{tabular}

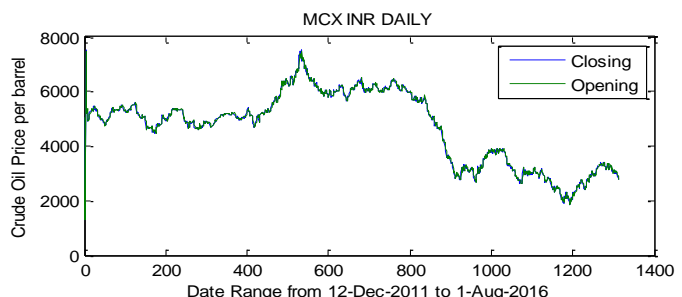

(a)

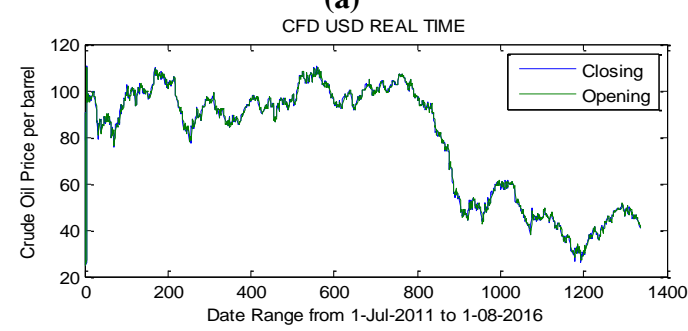

(b)

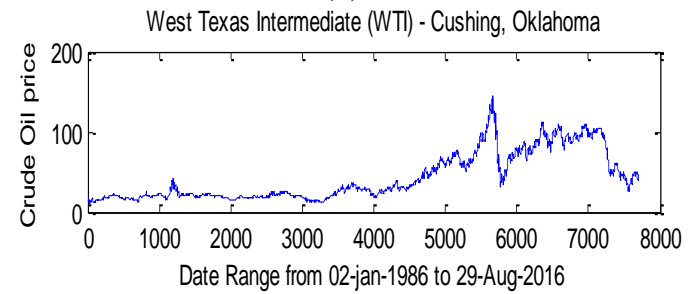

(c)

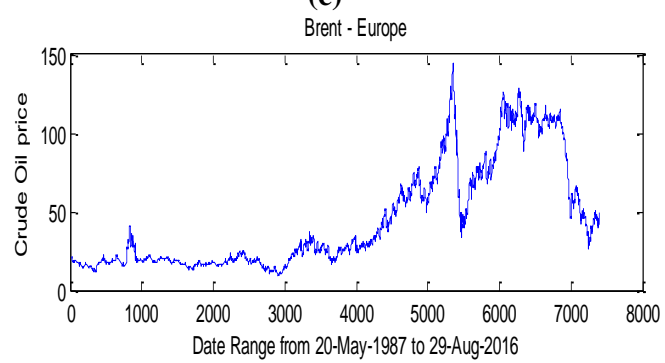

(d)

Fig. 1: Opening and closing indices graph of a) MCX INR Daily b) CFD USD Real Time c) WTI d) BRENT

\subsection{LBNF Model and Technical Indicators [35-37] For Building of Dataset}

Let $\mathrm{U}=\left\langle U_{o}, U_{l}, U_{h}, U_{c}>\right.$ represent data set where column $U_{o}=$ stock opening , $U_{l}=$ stock lower, $U_{h}=$ stock higher and $U_{c}=$ stock closing value for any day $u_{i}$ as shown in equation (1). Here $u_{i f} \in \mathfrak{R}^{n}$ for $f=\langle o, l, h, c\rangle=U$.

$$
U=\begin{gathered}
u_{1} \\
u_{2}
\end{gathered}\left(\begin{array}{cccc}
U_{o} & U_{l} & U_{h} & U_{c} \\
u_{2 o} & u_{1 l} & u_{1 h} & u_{1 c} \\
\vdots & u_{2 l} & u_{2 h} & u_{2 c} \\
u_{n o} & u_{n l} & u_{n h} & u_{n c}
\end{array}\right)
$$

Considering equation (1) as the given dataset, most of the prediction logic will try to predict data for different time series a) $u_{n+1}$ b) $u_{n+7}$ c) $u_{n+30}$ as one day, one week and one month in advance.

For prediction, classifier need to be trained which need input in the form of $\left(u_{i}, y_{i}\right)$. Where $y_{i}$ is the $i^{\text {th }}$ class label for any input feature $u_{i}$.Major issues related to any stock exchange data set is that it is unsupervised i.e., with no class label. Hence for identifying the class label for any feature we have built new technique called Look Back $N$ Feature (LBNF) algorithm.

Assume the task is to discover the class level $y_{i}$ of $u_{i}$ feature when $u_{1} u_{2}, \ldots, u_{i}, u_{i+1}$ is known. Looking towards the figures 2 (a) and (b), it is clearly revealed that the possibility of class level $y_{i}$ of $u_{i}$ can be any from the set $\{1,2,3,4\}$ and $\{1,2,3,4,5,6,7,8\}$ for $N=$ 1 and $N=2$ by Look back scheme respectively. In LBNF, $N$ represents the difference in level of current feature to the previous features (to be more specific $N$ is depth towards past from the current indices). In the figure 2 , each node depicts the feature, which has two possibilities that either the future will be profit 
represented by 1 or future will be loss represented by 0 . Now considering the node of interest $u_{i}$ for which $y_{i}$ need to be defined. With current scheme let say for $N=1$, our interest will be to know the previous state or feature $u_{i-1}$ has made loss or profit claiming to either get 0 or 1 string as past experience and just checking the next $u_{i+1}$ indices to find the future string of 0 or 1 . Note that for training set both past and future is available. Now with $N$ level look back scheme, total possibilities of binary string can be formed will be $\mathrm{L}$ given by equation (2).

$$
L=2^{N+1}
$$

Where, +1 is for just future indices. So, for $N=1$ we will have $L=$ 4 possibilities of binary string $\{00,01,10,11\}$, which leaves four choice for class label class level $y_{i}$ for $u_{i}$. For $N=2$, we will have $L=8$ possibilities of binary string $\{000,001,010,011,100,101$,
$110,111\}$ to act as class level class level $y_{i}$ for $u_{1}$. The more we choose the value of $N$ more refinement of class level will be seen. The following algorithm shows the steps to construct class label of the dataset. Algorithm can be called by sending parameter as opening or closing price as per user choice of interest, $u_{n}$ is the $n$th feature whose level is to be determined and $N$ is the depth in past.

\footnotetext{
Algorithm LBNF (price, $n, N$ )

Initialize binary_string <- empty

Step 1: $n<-$ feature index whose label is to be defined

Step 2: $N<$ - User choice of looking back (difference in feature indices)

Step 3: for index <- $(n-N)$ to $n$

binary_string <- binary_string $U$ price $($ index $)>$ price $($ index +1$)$ ? '0': ' 1 '

Step 4: $y(n)<-$ BinarytoDecimal(binary_string)
}

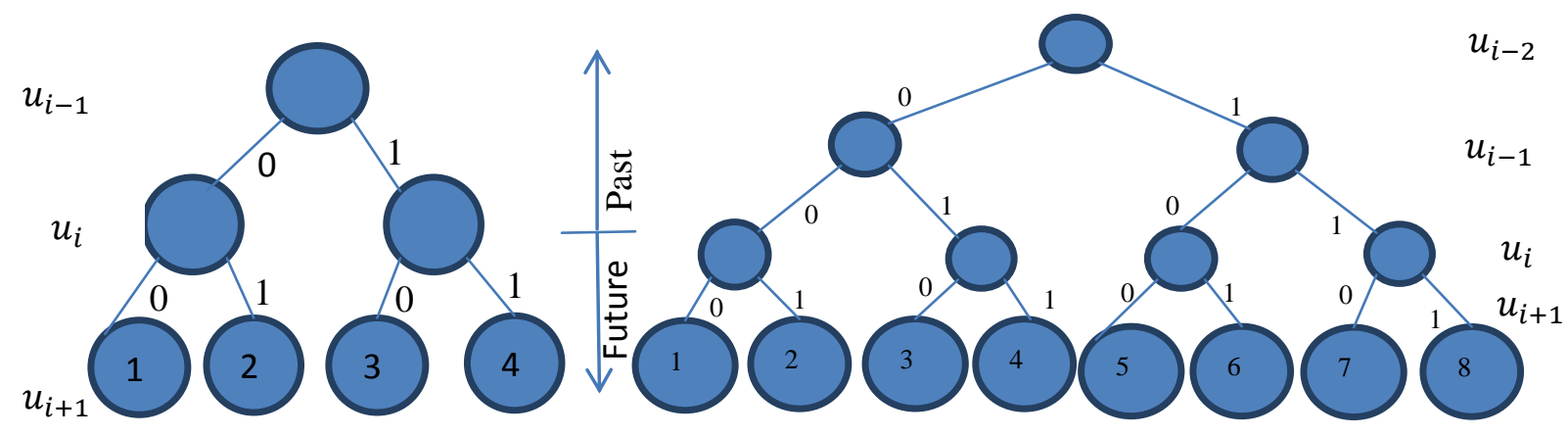

(a)

(b)

Fig. 2: Look Back binary tree for a) $N=1$ b) $N=2$, where $N$ is the difference in the number of level from current feature to previous features

For more smoothening the data set and have more grip over prediction few technical indicators such as Moving Average (MA), Relative Strength Index (RSI), Moving Average Convergence Divergence (MACD), William\%R, True Strength Index (TSI), and Volatility Ratio (VR) [35-37] are included and defined in the following equations ((3) to (8)).
Moving
Average (MA)

$$
\begin{aligned}
& \mathrm{MA}_{\mathrm{i}}=\frac{1}{2 \mathrm{~N}+1}(\mathrm{MA}(\mathrm{i}+\mathrm{N})+\mathrm{MA}(\mathrm{i}+\mathrm{N}-1)+ \\
& \operatorname{MA}(\mathrm{i}-\mathrm{N}))
\end{aligned}
$$
RSI

$$
\mathrm{RSI}=100-\frac{100}{1+\frac{\operatorname{EMA}(\mathrm{U}, \mathrm{n})}{\operatorname{EMA}(\mathrm{D}, \mathrm{n})}}
$$
MACD

$$
\mathrm{MMA}_{\mathrm{i}}=\frac{(\mathrm{N}-1) \mathrm{xMMA}_{\mathrm{i}-1}+\mathrm{u}_{\mathrm{ic}}}{\mathrm{N}}
$$

William\%R

$$
\% \mathrm{R}=\frac{\text { high }_{\mathrm{N} \text { days }}-\text { close }_{\text {today }}}{\text { high }_{\mathrm{N} \text { days }}-\text { low }_{\mathrm{N} \text { days }}} \times 100
$$

TSI

$$
\operatorname{TSI}\left(\mathrm{c}_{0}, \mathrm{r}, \mathrm{s}\right)=100 \times \frac{\operatorname{EMA}(\operatorname{EMA}(\mathrm{m}, \mathrm{r}), \mathrm{s})}{\operatorname{EMA}(\operatorname{EMA}(|\mathrm{m}|, \mathrm{r}), \mathrm{s})}
$$

Volatility Ratio

$$
\mathrm{VR}=\frac{\text { True Range }}{\text { True Range }_{\text {for last } \mathrm{n} \text { periods }}}
$$

$$
U=u_{2}\left(\begin{array}{ccccccccc}
U_{o} & U_{l} & U_{h} & U_{c} & \Delta c_{c} & T I_{1} & T I_{2} & T I_{6} & y \\
u_{1 o} & u_{1 l} & u_{1 h} & u_{1 c} & \Delta c_{c}^{1} & T I_{11} & T I_{12} & T I_{16} & y_{1} \\
u_{2 o} & u_{2 l} & u_{2 h} & u_{2 c} & \Delta c_{c}^{2} & T I_{21} & T I_{22} & T I_{26} & y_{2} \\
\vdots & \vdots & \vdots & \vdots & \vdots & \vdots & \vdots & \vdots & \vdots \\
u_{n o} & u_{n l} & u_{n h} & u_{n c} & \Delta c_{c}^{n} & T I_{n 1} & T I_{n 2} & T I_{n 6} & y_{n}
\end{array}\right)
$$

Where, $T I_{i}$ are the technical indicator discussed above. $\Delta c_{c}^{i}$ is the change in closing price of $i^{\text {th }}$ feature with $i+1$ feature. $\Delta c_{c}^{i}$ will help in predicting the price in advance will be discussed shortly in section 2.4 . 
$\Delta c_{c}^{i}=u_{i c}-u_{i+1 c}$

If $\Delta c_{c}^{i}>0$ then future will be loss and current feature $u_{i}$ will be linked with $u_{i+1}$ by binary string ' 0 ', else future will be profit and current feature $u_{i}$ will be linked with $u_{i+1}$ by binary string ' 1 ', as discussed in figure 2 .

For all the three time series data are first scaled between 0 and 1 using equation (11).

$\tilde{u}^{i j}=\frac{u_{i j}-u_{\min j}}{u_{\operatorname{maxj}}-u_{\min j}}$

Where $u_{i j}$ is the $j^{\text {th }}$ attribute value of $i^{\text {th }}$ feature $u_{i}$, and $u_{\min j}$ and $u_{\max j}$ are the $j^{\text {th }}$ minimum and maximum value of the data set respectively, and $\tilde{u}^{i}$ is the scaled price of $i^{\text {th }}$ day. The Mean Absolute Percentage Error (MAPE) and Root Mean Square Error (RMSE) [38] are used to measure the performance of the models. The MAPE and RMSE are defined as

$\mathrm{MAPE}=\frac{1}{T} \sum_{i=1}^{T}\left|\frac{d_{i}-\hat{d}_{i}}{d_{i}}\right| \times 100$

$\mathrm{RMSE}=\sqrt{\frac{1}{T}} \sum_{i=1}^{T}\left(d_{i}-\hat{d}_{i}\right)^{2}$

Where $T$ is the total number of testing data, $d_{i}$ and $\widehat{d}_{i}$ is the desired and predicted output respectively.

\subsection{P-Gram Sliding Window Technique [39] for Building Training and Testing Set}

Let there are $n$ features and 11 column vectors $\langle l, h, o, c, T 1, \ldots, T 7\rangle$ in the extended dataset $U$, where column feature $\Delta c$ and $y$ is used for evaluating future price and class label respectively. Training set is divided into $n-p$ set where $p$ the sliding window size is. Let for $p=3$ we will have training feature vectors as $T=\left\{\left(u_{1}, u_{2}, u_{3}\right),\left(u_{2}, u_{3}, u_{4}\right), \ldots,\left(u_{n-2}, u_{n-1}, u_{n}\right)\right\}$ and for every trining vector $\left(u_{i}, u_{i+1}, u_{i+2}\right)$ testing feature $S=$ $u_{i+3} \forall i=1, \ldots, n$. Now any classifier can be trained with $T_{j}$ and simultaneously can be tested with $S_{j} \forall j=1, \ldots, n-p$ and $T_{j} \in$ $T, S_{j} \in S$.

\subsection{Support Vector Machine and $K$-NN}

To extension of previous work [38], we fix the classifier SVM [15-18] for classification of time series feature as profit or loss and finding nearest neighbour from last $k^{\text {th }}$ feature which matches the prediction made by SVM. Finally we take the mean of prices results obtained from $k$-NN [38] as the final predicted closing or opening value of the time series dataset.

Given a training set with label pairs $\left(u_{i}, y_{i}\right), i=1, \ldots, n$ where $u_{i} \in \Re^{n}$ and $y \in$ class label as discussed in section 2.2, the SVM requires solution of the following optimization problem:

$\min \quad w, b, \xi \frac{1}{2} w^{T} w+C \sum_{i=1}^{n} \xi_{i}$

Subject to: $y_{i}\left(w^{T} \phi\left(u_{\mathrm{i}}\right)+\mathrm{b} \geq 1-\xi_{\mathrm{i}}, \xi_{\mathrm{i}} \geq 0\right.$.

Many times $u_{i}$ is non linear separable which found difficult to classify hence dataset is mapped into higher dimension by using kernel function $k\left(u_{i}, u_{j}\right) \equiv \Phi\left(u_{i}\right)^{T} \Phi\left(u_{j}\right)$. SVM is trained using different kernels [38] like,

a) Linear kernel: $\left(u_{i}, u_{j}\right)=u_{i}^{T} u_{j}$,

b) Polynomial kernel: $k\left(u_{i}, u_{j}\right)=\left(\gamma u_{i}^{T} u_{j}+r\right)^{d}, \gamma>0$, and c) Radial Basis kernel (RBF) $k\left(u_{i}, u_{j}\right)=\exp \left(-\gamma \| u_{i}-\right.$ $\left.u_{j} \|^{2}\right), \gamma>0$.

Here, $C, \gamma, r$, and $d$ are kernel parameters which are initialized depending on the dataset. The choice of best kernel parameter is affected by the size of training set. In this paper we have used $\mathrm{RBF}$. The data sets with various combination of parameters $\{\mathrm{C}, \gamma\}$ has been implemented, in which the parameter $C$ is chosen from $\left\{2^{-5}, 2^{-4}, \ldots, 2^{5}\right\}$ and $\gamma$ from $\left\{2^{-15}, 2^{-14}, \ldots, 2^{-1}\right\}$.

Now the $k$-nearest point is mined using $k$-NN based on $y_{i}$ predicted by SVM from the past $k$ datasets. Identification of $k$ different feature from the query point is done by measuring the distance using following equation (18).

$D(x, p)= \begin{cases}\sqrt{(x-p)^{2}} & \text { Euclidean } \\ (x-p)^{2} & \text { Euclidean squared } \\ A b s(x-p) & \text { Cityblock } \\ \operatorname{Max}(|x-p|) & \text { Chebyshev }\end{cases}$

Where $x, p$ and $D$ are the query point, a case from the examples sample, and distance between $x$ to $p$ respectively.

\section{Proposed Model for Crude Oil Prediction}

Our proposed model as shown in figure 3 is divided into three phases. In phase 1 , first look back $N$ feature algorithm is used to evaluate class label for each features, second technical indicators are used to smoothen the dataset, and third change in closing price is evaluated. In phase 2 , data is split into $P$ gram feature vector as discussed in section 2 for training and testing of SVM classifier. The class label $r$ predicted by classifier is compared with class label $y$ to compute accuracy of our model. In phase 3, from past data $k$-nearest neighbor search is made whose class label is equal to $r$ and mean of those $k$-nearest neighbor's $\Delta c$ is evaluated. Result is then added with current closing value as the predicted value in advance.

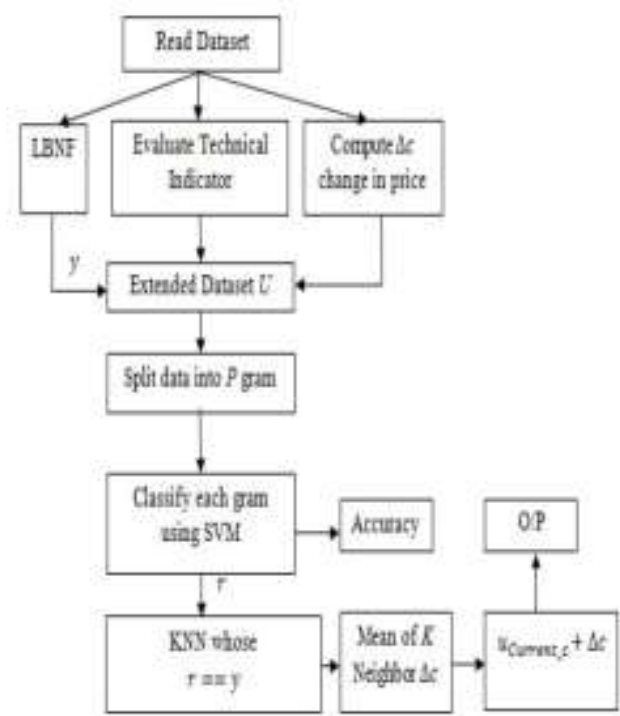

Fig. 3: Proposed look back $N$ feature model

\section{Experimental Evaluation and Result Analysis}

The experiment undertaken in this chapter has taken into account proposed model, four datasets and three time horizons. The empirical results have been presented in terms of target vs. predicted values and error convergence speed. A comparative analysis of the performance of all the models along with the time 
horizons has also been presented in this paper.

The experimental data obtained from two significant crude oil dataset discussed in section 2 are used to predict the indices 1 day, 1 week and 1 month in advance. Proposed model discussed in previous section consist of few parameters such as value for $N$ in LBNF algorithm, window size $p$, which need to tune properly. With hit and trial method we have tested our proposed work with $N=1,2,3,4$ and 5 and $p=10$ to 100 , it is found that for $N=3$ and $p=60$, SVM classifier gives optimal result for MCX INR daily dataset shown in table 2 . Similarly for CFD USD real time dataset optimal value for $N$ and $p$ better accuracy achieved are 3 and 50 respectively. Looking to the graph in figure 4 and figure 5 , it can be understood that with increase in value of $p$ from 10 to 100 the accuracy curve was rising till mid of the graph 60 (for MCX INR Daily dataset) and 50 (CFD USD real time dataset) respectively and later accuracy falls down. Thus, giving a maximum accuracy of $89.6 \%$ and $90.3 \%$ for both the dataset. Figure 6-7 show the comparison of target vs. predicted stock prices during testing of two datasets (MCX INR and CFD USD real time) for 1 day, 1 week and 1 month in advance.

Similarly for other two dataset WTI and BRENT the optimum value of parameter $\mathrm{N}, p$ is shown in table 3. The list of parameter for SVM classifier and $k$-NN search for data set is shown in table 4. Figure 8 shows the crude oil prediction curve in comparison with original in all the three time series daily, weekly and monthly. From the figure it can be noticed that it is hard to differentiate the original and predicted curve for which small zoom in figure is embedded with the figure stating the resultant difference in prediction vs. original graph.

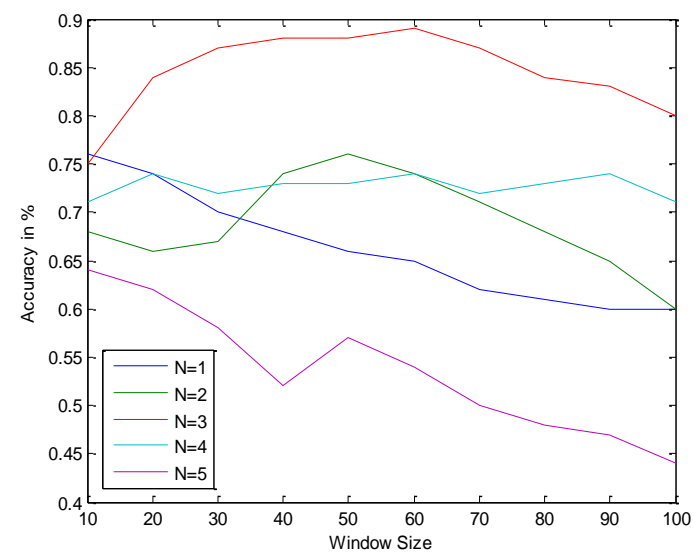

Fig. 4: Accuracy curve against window size and value for $\mathrm{N}$ in LBNF for MCX INR DAILY dataset

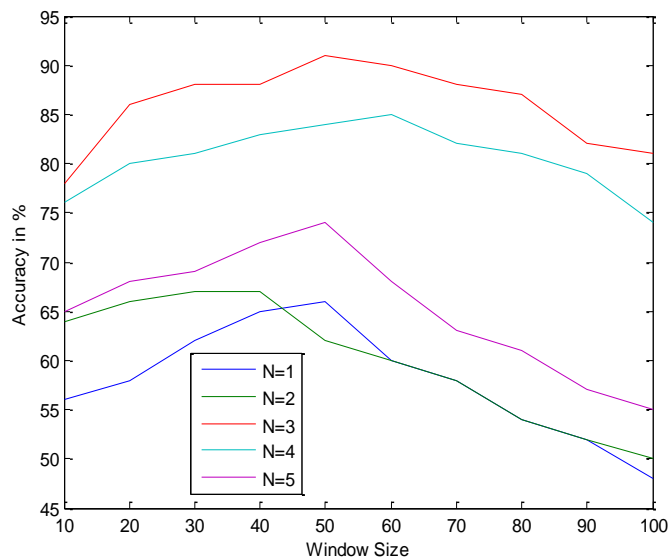

Fig. 5: Accuracy curve against window size and value for $\mathrm{N}$ in $\mathrm{LBNF}$ for CFD USD REAL TIME

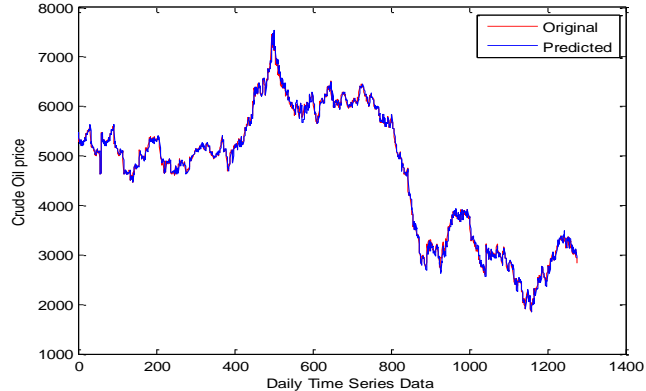

(a)

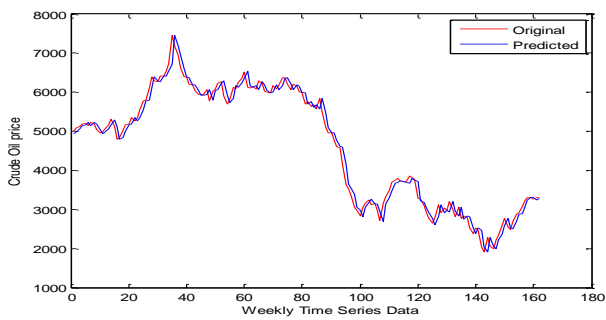

(b)

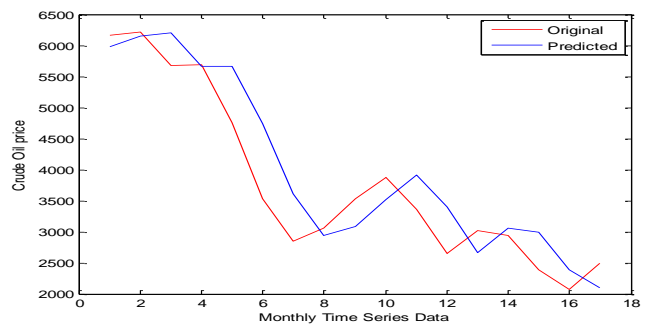

(c)

Fig. 6: Crude Oil Price Predicted vs Original for MCX INR DAILY dataset a) Daily b) Weekly c) Monthly

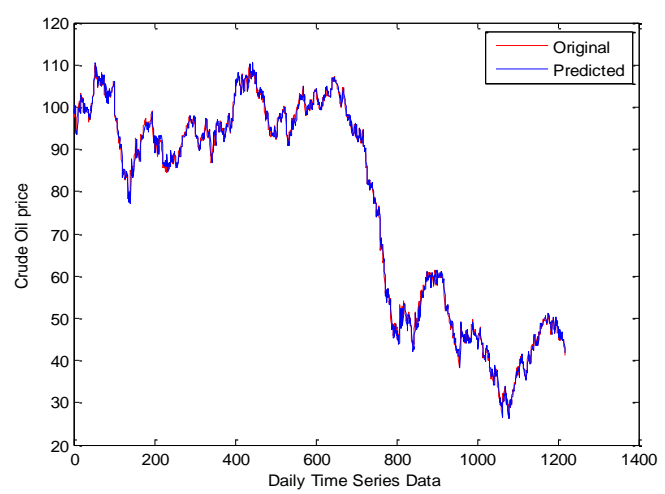

(a)

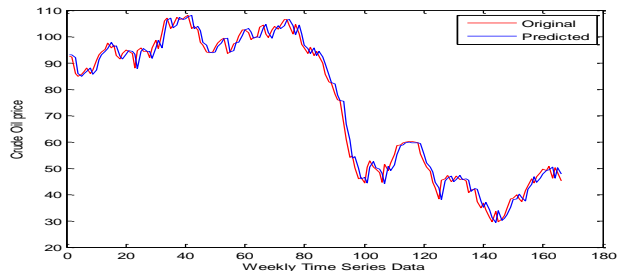

(b)

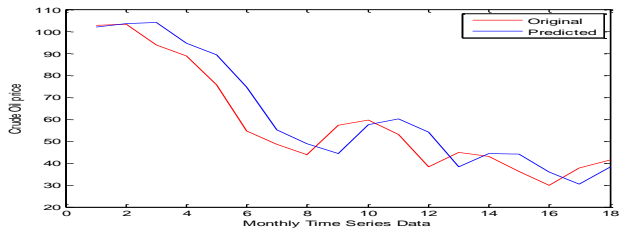

(c)

Fig. 7: Crude Oil Price Predicted vs Original for CFD USD REAL TIME a) Daily b) Weekly c) Monthly 
Table 2: Optimum value of parameter $N$ in LBNF and $p$ window size for MCX INR and CFD USD dataset

\begin{tabular}{|l|l|l|l|}
\hline Dataset & $\boldsymbol{N}$ in LBNF & Window size $\boldsymbol{p}$ & Accuracy in \% \\
\hline MCX INR & 3 & 60 & 89.60 \\
\hline CFD USD & 3 & 50 & 90.30 \\
\hline
\end{tabular}

Table 3: Optimum value of parameter $N$ in LBNF and $p$ window size for BRENT and WTI dataset

\begin{tabular}{|l|l|l|l|}
\hline Dataset & $\boldsymbol{N}$ in LBNF & Window size $\boldsymbol{p}$ & Accuracy \\
\hline BRENT & 3 & 70 & 88.23 \\
\hline WTI & 3 & 50 & 82.40 \\
\hline
\end{tabular}

Table 4: Range of value for SVM and KNN parameter

\begin{tabular}{|c|c|c|c|c|c|c|}
\hline Data Set & \multicolumn{6}{|c|}{ Parameter Value } \\
\hline & \multicolumn{2}{|l|}{1 Day } & \multicolumn{2}{|l|}{1 Week } & \multicolumn{2}{|l|}{1 Month } \\
\hline & $\operatorname{SVM}\{C, \gamma\}$ & $\mathrm{k}$ & $\operatorname{SVM}\{C, \gamma\}$ & $\mathrm{k}$ & $\mathrm{SVM}\{\mathrm{C}, \gamma\}$ & $\mathrm{K}$ \\
\hline MCX INR Daily & $\left\{2^{-4}, 2^{-15}\right\}$ & 10 & $\left\{2^{-4}, 2^{-4}\right\}$ & 10 & $\left\{2^{-3}, 2^{-1}\right\}$ & 5 \\
\hline CFD USD Real Time & $\left\{2^{-2}, 2^{-10}\right\}$ & 10 & $\left\{2^{-2}, 2^{-12}\right\}$ & 10 & $\left\{2^{-3}, 2^{-2}\right\}$ & 5 \\
\hline BRENT & $\left\{2^{-2}, 2^{-10}\right\}$ & 10 & $\left\{2^{-2}, 2^{-12}\right\}$ & 10 & $\left\{2^{-3}, 2^{-2}\right\}$ & 5 \\
\hline WTI & $\left\{2^{-4}, 2^{-15}\right\}$ & 10 & $\left\{2^{-4}, 2^{-4}\right\}$ & 10 & $\left\{2^{-3}, 2^{-1}\right\}$ & 5 \\
\hline
\end{tabular}

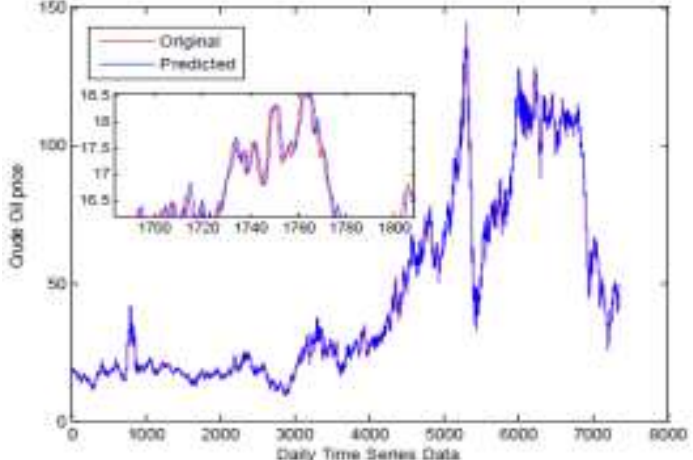

(a)

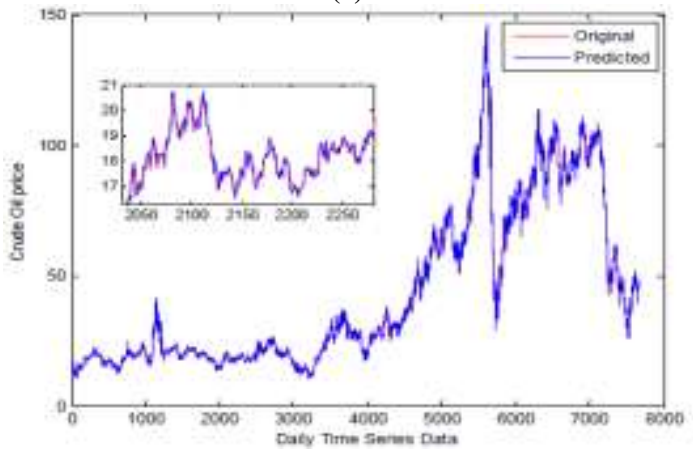

(b)

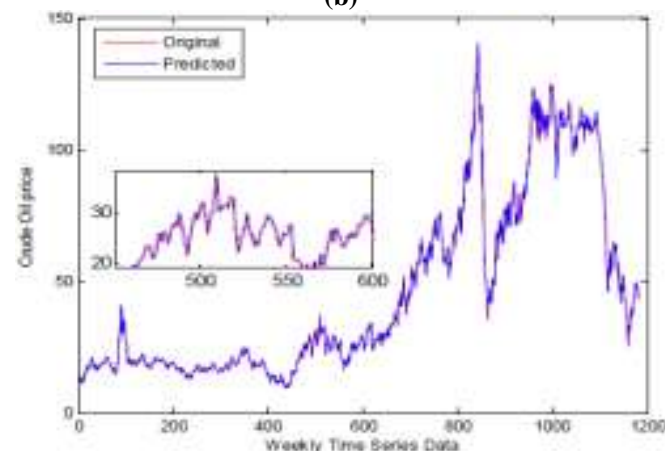

(c)

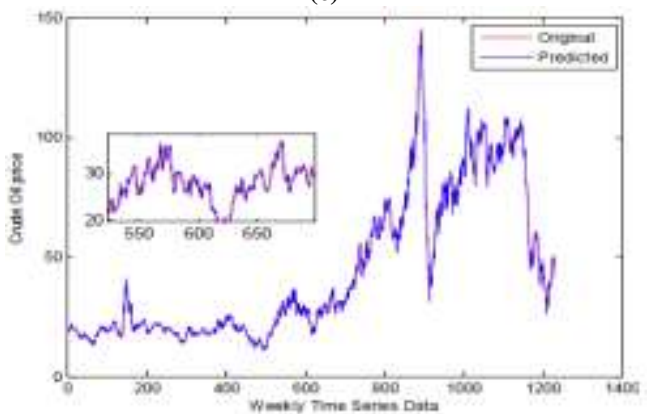

(d)

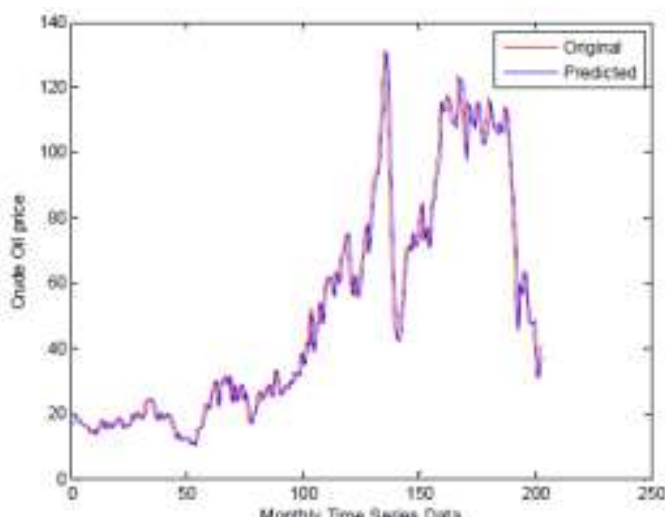

(e)

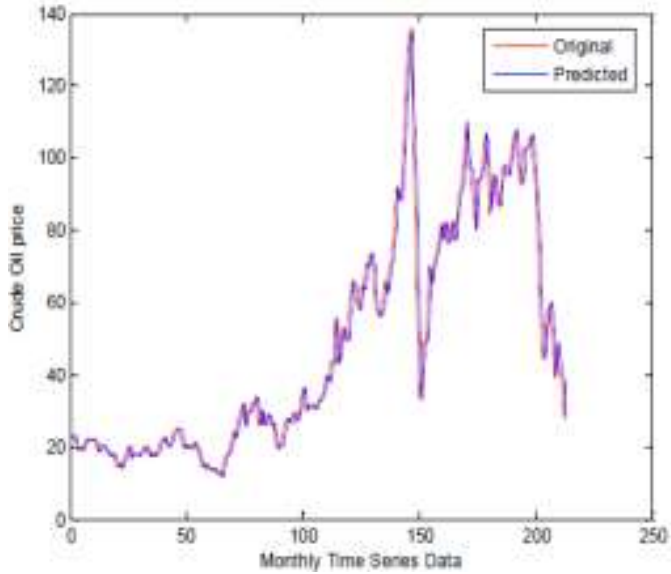

(f)

Fig. 8: Crude Oil Prices: BRENT - Europe Original vs prediction on different time series (a) Daily (c) Weekly (e) Monthly, and Crude Oil Prices: West Texas Intermediate (WTI) - Cushing, Oklahoma Original vs Prediction time series (b) Daily (d) Weekly (f) Monthly.

We have compared our model with different model for Brent and WTI dataset, but with unavailability of a good research article for MCX and CFD dataset we were not able to compare. Table 5 shows the comparison of our proposed work with others for WTI and BRENT dataset. Proposed method has lower RMSE of 0.1333 and 0.1253 which is almost $70 \%$ better than Grey wave forecasting and ARMA model.

Table 5: Comparison of the Forecasting Accuracy Using Different Prediction Techniques

\begin{tabular}{|l|l|l|}
\hline Data Set & Methods & RMSE \\
\hline WTI & Grey Wave Forecasting[29] & 0.2006 \\
\hline & ARMA(1,1)[28] & 0.2058 \\
\hline & Proposed & $\mathbf{0 . 1 3 3 3}$ \\
\hline BRENT & Grey Wave Forecasting[29] & 0.1987 \\
\hline & ARMA $(1,1)[28]$ & 0.2228 \\
\hline & Proposed & $\mathbf{0 . 1 2 5 3}$ \\
\hline
\end{tabular}




\section{Conclusion}

This paper proposes a unique dimension of class division from just profit and loss ( 2 class problem) to $n$ class problem, depending upon the choice of user to identify how long he will look back from particular instance of time for prediction of crude oil price in next time frame. This research concentrates on a new concept of shifting the dataset into $n$ class problem and thus giving more scope to classifier to achieve better training and accuracy. By result analysis on four datasets, it can be said that proposed method can be used for crude oil prediction though the value of $N$ for look back $N$ feature is still user defined. In future we will try to define any heuristic way to figure out the value of $N$ so that an optimum result can be obtained.

\section{References}

[1] Natural resources - definition of natural resources in English, Oxford Dictionaries. 2014-04-20. Retrieved 2016-12-12. https://en.wikipedia.org/wiki/Natural_resource.

[2] Guerriero V, Mazzoli S, Iannace A, Vitale S, Carravetta A \& Strauss C, "A permeability model for naturally fractured carbonate reservoirs", Marine and Petroleum Geology, Vol. 40, (2013), pp. $115-134$

[3] Azoff EM, Neural network time series forecasting of financial markets, John Wiley \& Sons, Inc. (1994).

[4] Haidar I, Kulkarni S \& Pan H, "Forecasting model for crude oil prices based on artificial neural networks", International Conference on Intelligent Sensors, Sensor Networks and Information Processing, (2008), pp. 103-108.

[5] Haykin S, Neural networks, a comprehensive foundation, (1994).

[6] Yu L, Wang S \& Lai KK, "Forecasting crude oil price with an EMD-based neural network ensemble learning paradigm", Energy Economics, Vol. 30, No. 5, (2008), pp. 2623-2635.

[7] Kulkarni S \& Haidar I, "Forecasting model for crude oil price using artificial neural networks and commodity futures prices", arXiv preprint arXiv: 0906.4838, (2009).

[8] Pan H, Haidar I \& Kulkarni S, "Daily prediction of short-term trends of crude oil prices using neural networks exploiting multimarket dynamics", Frontiers of Computer Science in China, Vol. 3, No. 2, (2009), pp. 177-191.

[9] Abdullah SN \& Zeng X, "Machine learning approach for crude oil price prediction with Artificial Neural Networks-Quantitative (ANN-Q) model", The 2010 International Joint Conference on Neural Networks (IJCNN), (2010), pp. 1-8.

[10] Chen X \& Qu Y, "A prediction method of crude oil output based on artificial neural networks", Proc. of the IEEE Int. Conf. Comput. Inf. Sci, (2011), pp. 702-704.

[11] Gunawan R, Khodra ML, "Commodity price prediction using neural network case study: Crude palm oil price", International Conference on Computer, Control, Informatics and Its Applications (IC3INA), (2013), pp. 243-248.

[12] Gunawan R \& Khodra ML, "Commodity price prediction using neural network case study: Crude palm oil price", International Conference on Computer, Control, Informatics and Its Applications (IC3INA), (2013), pp. 243-248.

[13] Xu D, Zhang Y, Cheng $\mathrm{C}$, Xu W \& Zhang L, "A neural networkbased ensemble prediction using PMRS and ECM", 47th Hawaii International Conference on System Sciences, (2014), pp. 13351343.

[14] Abubakar AI, Chiroma H \& Abdulkareem S, “Comparing performances of neural network models built through transformed and original data", International Conference on Computer, Communications, and Control Technology, (2015), pp. 364-369.

[15] Xie W, Yu L, Xu S \& Wang S, "A new method for crude oil price forecasting based on support vector machines", International Conference on Computational Science, (2006), pp. 444-451.

[16] Bao Y, Yang Y, Xiong T \& Zhang J, "A comparative study of multistep-ahead prediction for crude oil price with support vector regression", Fourth International Joint Conference on Computational Sciences and Optimization (CSO), (2011), pp. 598602.

[17] Khashman A \& Nwulu NI, "Intelligent prediction of crude oil price using Support Vector Machines", IEEE 9th International
Symposium on Applied Machine Intelligence and Informatics (SAMI), (2011), pp. 165-169.

[18] Fan L, Pan S, Li Z \& Li H, "An ICA-based support vector regression scheme for forecasting crude oil prices", Technological Forecasting and Social Change (2016).

[19] Behmiri NB \& Manso JRP, "Crude oil price forecasting techniques: a comprehensive review of literature", CAIA Alternative Investment Analyst Review, Vol. 2, No. 3, (2013), pp. 30-48.

[20] Yu L, Dai W \& Tang L, "A novel decomposition ensemble model with extended extreme learning machine for crude oil price forecasting", Engineering Applications of Artificial Intelligence, Vol. 47, (2016), pp. 110-121.

[21] Yu L, Wang S, Wen B \& Lai KK, "An AI-agent-based trapezoidal fuzzy ensemble forecasting model for crude oil price prediction", $3 r d$ International Conference on Innovative Computing Information and Control, IEEE, (2008), pp. 327-327.

[22] Behmiri NB, Manso JRP, "Crude Oil Price Forecasting Techniques: A Comprehensive Review of Literature" (2013).

[23] He Angela WW, Kwok Jerry TK \& Wan Alan TK, "An empirical model of daily highs and lows of West Texas Intermediate crude oil prices", Energy Economics, Vol. 32, No. 6, (2010), pp. 1499-1506.

[24] Ekaterini P \& Theologos P, "Speculative behaviour and oil price predictability", Economic Modelling. Vol. 47, (2015), pp. 128-136.

[25] Atilim M \& Ekin T, "Forecasting oil price movements with crack spread futures", Energy Economics, Vol. 31, No. 1, (2009), pp. 8590.

[26] Lean Y, Shouyang W \& Keung LK, "Forecasting crude oil price with an EMD-based neural network ensemble learning paradigm", Energy Economics, Vol. 30, No. 5, (2008), pp. 2623-2635.

[27] Kaijian H, Lean Y \& Keung LK, "Crude oil price analysis and forecasting using wavelet decomposed ensemble model", Energy. 2012, Vol. 46, No. 1, (2012), pp. 564-574.

[28] Ziran L, Jiajing S \& Shouyang W, "An information diffusion-based model of oil futures price", Energy Economics, Vol. 36, (2013), pp. 518-525.

[29] Chen Y, Zou Y, Zhou Y \& Zhang C, "Multi-step-ahead Crude Oi Price Forecasting based on Grey Wave Forecasting Method", Procedia Computer Science, Vol. 91, (2016), pp. 1050-1056.

[30] Van Der Walt C \& Barnard E, "Data characteristics that determine classifier performance", (2006).

[31] http://in.investing.com/commodities/crude-oil

[32] Manescu C \& Robays IV, "Forecasting the Brent oil price: addressing time-variation in forecast performance", (2014).

[33] Chiroma H, Abdulkareem S \& Herawan T, "Evolutionary Neural Network model for West Texas Intermediate crude oil price prediction”, Applied Energy, Vol. 142, (2015), pp. 266-273.

[34] Beckers B, "Forecasting the Nominal Brent Oil Price with VARsOne Model Fits All?", International Monetary Fund, (2015).

[35] Magda B \& Fayek, "Multi-objective optimization of technical stock market indicators using gas", Int. J. Comput. Appl, 0975-8887, Vol. 68, No. 20, (2013).

[36] http://stockcharts.com/school/doku.php?id=chart school:technicalindicators:williams $r$

[37] Moldovan D, Moca M \& Nitchi S, "A stock trading algorithm model proposal, based on technical indicators signals", Informatica Economica, Vol. 15, No. 1, (2011), pp. 183

[38] Nayak RK, Mishra D \& Rath AK, "A Naïve SVM-KNN based stock market trend reversal analysis for Indian benchmark indices", Applied Soft Computing, Vol. 35, (2015), pp. 670-680.

[39] Jayasinghe GK, Culpepper JS \& Bertok P, "Efficient and effective realtime prediction of drive-by download attacks", Journal of Network and Computer Applications, Vol. 38, (2014), pp. 135-149. 